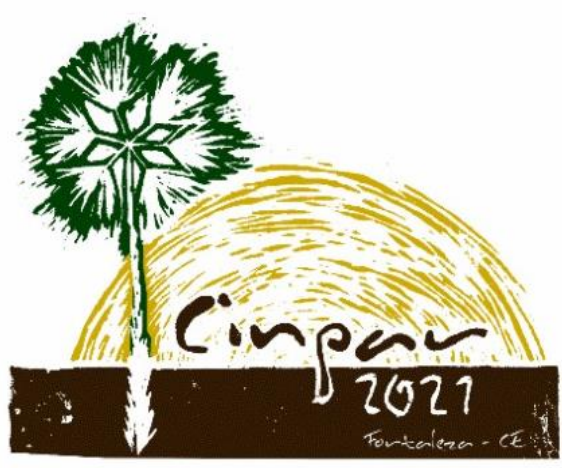

XVII Congresso Internacional sobre Patologia e

Reabilitação das Construções

XVII Congreso Internacional sobre Patología y Rehabilitación de las Construcciones

XVII International Conference on Pathology and Constructions Rehabilitation

FORTALEZA (Brasil), 3 a 5 de junho de 2021

https://doi.org/10.4322/CINPAR.2021.041

\title{
Avaliação da resistência do concreto por meio do ensaio de ultrassonografia
}

\section{Evaluation of concrete strength by ultrasound test} \author{
MARTINI $^{4}$, Thiago Pena BORTONE ${ }^{5}$, Marcos de Paulo RAMOS ${ }^{6}$ \\ ${ }^{1}$ CEFET-MG, Curvelo, Brasil, gabrielaandrade74@hotmail.com \\ ${ }^{2}$ CEFET-MG, Curvelo, Brasil, anapaulapaisgott@hotmail.com \\ ${ }^{3}$ CEFET-MG, Curvelo, Brasil, laurakaddyjean@ymail.com \\ ${ }^{4}$ CEFET-MG, Curvelo, Brasil, martini@cefetmg.br \\ ${ }^{5}$ CEFET-MG, Curvelo, Brasil, thiago.bortone@cefetmg.br \\ ${ }^{6}$ CEFET-MG, Curvelo, Brasil, marcos.ramos@cefetmg.br
}

Gabriela Santos DE ANDRADE ${ }^{1}$, Ana Paula Pais GOTT ${ }^{2}$, Laura Kaddyjean Oliveira SOARES ${ }^{3}$, Rachel Jardim

Resumo: Ao longo dos anos, existiu uma ideia de que as estruturas de concreto armado eram eternas, ou seja, à prova de deterioração. Sendo assim, hoje é uma máxima de que tais estruturas também se deterioram, situação evidenciada pelo estado em que algumas obras de arte rodoviárias apresentam-se.O método tradicional de investigação que consiste na extração de amostras de concreto da estrutura, e a posterior execução de ensaios de compressão axial, apresentam como desvantagens ser onerosa, além da restrição dos locais de retirada dos testemunhos, para que não se comprometa a estabilidade da estrutura. Em razão disso, os corpos-de-prova, em geral, são extraídos apenas das lajes em números relativamente pequenos, o que não permite um completo mapeamento dos níveis de resistência do concreto da estrutura.Neste contexto estão inseridos os ensaios não destrutivos (END) que viabilizam a coleta de dados sem a necessidade de extração de amostras, de maneira a causar pouco ou nenhum prejuízo. Para a avaliação de estruturas de concreto armado, estes ensaios auxiliam na caracterização e identificação de manifestações patológicas, além de monitorar alguns parâmetros físicos que estão relacionados aos processos de deterioração ou risco à estrutura. $O$ ensaio de ultrassom caracteriza-se num método não destrutivo que tem por objetivo a deteç̧ão de defeitos ou descontinuidades internas presentes nos mais variados tipos de materiais. Adicionalmente, possibilita medir a profundidade de fissuras, acompanharem a modificação de propriedades do concreto ao longo do tempo.O objetivo desse trabalho foi inspecionar os corpos de prova cilíndricos de concreto simples, de mesma composição de materiais, a fim de se avaliar a uniformidade das peças e realizar uma correlação entre os resultados dos ensaios de ultrassonografia com os resultados de ensaios destrutivos de resistência à compressão para diferentes idades. Os resultados apresentam um bom índice de correlação $\left(R^{2}=0.86\right)$ para os casos estudados.

Palavras-chave: ultrassom, ensaios não destrutivos, concreto.

Abstract: Over the years, there has been an idea that reinforced concrete structures were eternal, that is, deteriorating. Thus, today it is a maxim that such structures also deteriorate, a situation evidenced by the state in which some road works of art are presented.

The traditional method of investigation, which consists in the extraction of concrete samples from the structure, and the subsequent execution of axial compression tests, have as disadvantages to be costly, in addition to the restriction of the places of removal of the testimonies, so that the stability of the structure is not compromised. As a result, specimens are generally extracted only from slabs in relatively small 
numbers, which does not allow a complete mapping of the resistance levels of the concrete of the structure.

In this context, non-destructive tests (END) are inserted, which enable data collection without the need for sample extraction, in order to cause little or no damage. For the evaluation of reinforced concrete structures, these tests assist in the characterization and identification of pathological manifestations, in addition to monitoring some physical parameters that are related to the processes of deterioration or risk to the structure.

The ultrasound test is characterized by a non-destructive method that aims to detect defects or internal discontinuities present in the most varied types of materials. Additionally, it makes it possible to measure the depth of cracks, to monitor the change in concrete properties over time.

The objective of this work is to inspect cylindrical specimens of simple concrete, with the same composition of materials, in order to evaluate the uniformity of the parts and perform a correlation between the results of the ultrasound tests with the results of destructive tests of resistance to compression for different ages. The results show a good correlation index $\left(R^{2}=0.86\right)$ for the cases studied.

Keywords: ultrasound, non-destructive testing, concrete.

\section{Introdução}

O concreto teve seu uso amplamente difundido no último século em construções de pequeno, médio e grande porte, tais como residências, edifícios, pavimentos, estruturas de contenção, fundações, entre outras (MEDEIROS, 2007). Esse crescimento acelerado da construção civil provocou a necessidade de inovações tecnológicas que trouxeram aceitação de maiores riscos e consequentemente o desenvolvimento da indústria da construção civil (SOUZA e RIPPER, 1998).

De modo habitual, são feitos ensaios de resistência à compressão aos 28 dias em corpos de prova (CPs) cilíndricos (ou cúbicos) para verificar se os parâmetros mecânicos do concreto utilizado estão de acordo com os parâmetros exigidos em projeto. No entanto, os CPs não conseguem caracterizar, de maneira fiel, o concreto existente na estrutura, devido às diferentes condições de lançamento, compactação e condições de cura (EVANGELISTA, 2002).

Nas décadas de 70 e 80 houve um aumento nos estudos dos Ensaios Não Destrutivos (ENDs), com a intenção de melhor representar as características do concreto na própria estrutura, já que os ensaios padrão em corpos de prova representavam apenas a resistência à compressão do mesmo, em condições diferentes da estrutura real (CASTRO, 2009).

Ensaios de caráter não destrutivo se tornaram viáveis e adequados para auxiliar na avaliação do desempenho de estruturas em serviço. Quando se precisam obter dados de resistência de tais estruturas, o mais comum é recorrer à extração de corpos de prova para posterior análise em laboratório, através do rompimento destes em ensaio de compressão. O inconveniente deste tipo de avaliação é que muitas vezes, quando se está extraindo o testemunho, este se quebra num comprimento menor que o desejado, o que provoca, muitas vezes, a realização de novos furos e assim, deixando a estrutura danificada, sendo necessários reparos posteriores (MENEGHETTI, 1999).

De modo particular, o ultrassom é uma técnica de END que possui bom desempenho quando utilizado na determinação da resistência à compressão do concreto porém, devido às grandes diferenças na matriz constituinte do concreto, que ocorrem logo nas primeiras idades de um corpo de prova, oriundas do processo de hidratação do cimento, a umidade provoca mudanças significativas na velocidade de propagação das ondas ultrassônicas (MENEGHETTI, 1999).

Neste trabalho, faz-se uma proposta de avaliação da resistência à compressão axial do concreto utilizando o método da ultrassonografia. Para atingir tal fim, pretende-se realizar uma correlação entre os resultados dos ensaios de ultrassonografia com os resultados de ensaios destrutivos convencionais de resistência à compressão axial em diversas idades. 


\section{Definição de ensaio de ultrassom}

O ensaio de ultrassom permite que uma onda ultrassônica seja projetada em um material, sendo medida a velocidade de propagação de ondas ultrassônicas da mesma. Esta velocidade vai depender da natureza do material (sólido, homogêneo e isotrópico), da sua porosidade, propriedades elásticas, da presença de vazios ou de água no sistema de poros, entre outros fatores. A velocidade para meios sólidos, elásticos e homogêneos é dada pela Equação 1 e conseguinte as Equações 2 e 3 (LORENZI et al., 2008; MALHOTRA; CARINO, 1991).

$$
\begin{gathered}
V=\sqrt{\frac{K E_{d}}{\rho}} \\
K=\frac{(1-v) \cdot g}{(1+v)(1-v)} \\
E_{d}=\frac{V^{2} \rho}{K}
\end{gathered}
$$

Onde:V: Velocidade de Propagação de onda ultrassônica;

$E_{d}$ : Módulo de Elasticidade Dinâmico do material;

$\rho:$ densidade do material;

g: aceleração devido à gravidade;

v: Coeficiente de Poisson dinâmico do material.

As relações entre Módulos de Elasticidade Dinâmico (MOD) e Estático (MOE) com relação à Velocidade do Pulso Ultrassônico (VPU) são fornecidas na Tabela 1 e se aplicam aos concretos feitos com os tipos mais comuns de agregados naturais: areia, brita e cascalho. O Coeficiente de Poisson do concreto situa-se na faixa de 0,15 a 0,25 para a maioria dos concretos. No caso da determinação do Módulo de Elasticidade Dinâmico, assumindo-se um valor médio para o coeficiente de Poisson, o erro permanecerá na casa de $10 \%$ ou menos (COSTA, 2004; BS 1881 (BSI, 1986)).

Tabela 1 - Relação empírica entre Módulos de Elasticidade Estáticos e Dinâmicos e Velocidade de Pulso Ultrassônico

\begin{tabular}{|c|c|c|}
\hline $\begin{array}{c}\text { Velocidade } \\
\text { do pulso } \\
(\mathrm{km} / \mathrm{s})\end{array}$ & $\begin{array}{c}\text { MOD } \\
\left(\mathrm{MN} / \mathrm{m}^{2}\right)\end{array}$ & $\begin{array}{c}\mathrm{MOE} \\
\left(\mathrm{MN} / \mathrm{m}^{2}\right)\end{array}$ \\
\hline 3.6 & 24.000 & 13.000 \\
\hline 3.8 & 26.000 & 15.000 \\
\hline 4.0 & 29.000 & 18.000 \\
\hline 4.2 & 32.000 & 22.000 \\
\hline
\end{tabular}

\begin{tabular}{|c|c|c|}
\hline $\begin{array}{c}\text { Velocidade } \\
\text { do pulso } \\
(\mathrm{km} / \mathrm{s})\end{array}$ & $\begin{array}{c}\mathrm{MOD} \\
\left(\mathrm{MN} / \mathrm{m}^{2}\right)\end{array}$ & $\begin{array}{c}\mathrm{MOE} \\
\left(\mathrm{MN} / \mathrm{m}^{2}\right)\end{array}$ \\
\hline 4.4 & 36.000 & 27.000 \\
\hline 4.6 & 42.000 & 34.000 \\
\hline 4.8 & 49.000 & 43.000 \\
\hline 5.0 & 58.000 & 52.000 \\
\hline
\end{tabular}

Fonte: BS 1881(BSI, 1986)

De acordo com a NBR 8802 (ABNT, 2019), o ensaio de ultrassom consiste na medição, por meio eletrônico, do tempo de propagação de ondas ultrassônicas através do concreto, entre o emissor e o receptor. Um pulso de onda ultrassônica através do concreto é criado em um ponto na superfície do objeto teste e assim é medido o tempo de sua viagem desse ponto para outro. O comprimento percorrido entre os transdutores dividido pelo tempo de propagação resulta na velocidade média de propagação da onda ultrassônica, conforme a Equação 4:

$$
V=\frac{L}{t}
$$

Onde:

V: Velocidade de Propagação da onda ultrassônica;

Avaliação da resistência do concreto por meio do ensaio de ultrassonografia 
L: a medida da distância entre os eixos e /ou as faces dos transdutores;

t: tempo decorrido desde a emissão da onda até a sua recepção.

De acordo com Lorenzi et al. (2008), o ensaio pode também ser usado para entender a relação entre a qualidade do concreto, avaliada pela VPU, com sua resistência à compressão axial. Hipoteticamente, é possível estabelecer uma relação de regressão entre a densidade do concreto e a resistência à compressão axial. A oportunidade de estimar a resistência a partir de um ensaio rápido e não destrutivo é muito atraente, pois isto permitiria obter uma maior segurança quanto às características reais do concreto. Sendo assim, a ideia é explorar o fato de que a velocidade é uma função da densidade do material e de sua dureza, que estão correlacionadas com a resistência à compressão axial.

\section{Método de geração e recepção das ondas ultrassônicas}

A maior parte dos aparelhos para o ensaio de ultrassom identificam descontinuidades através da monitoração das reflexões sônicas transmitidas ao material através de um cabeçote acoplado na peça (SANTIN, 2003).

As ondas ultrassônicas são originadas a partir de um transdutor eletroacústico que, ligado a um circuito eletrônico, recebe um estímulo para variar mecanicamente, em uma frequência que pode ser de $20 \mathrm{kHz}$ até aos $200 \mathrm{kHz}$ conforme o transdutor. Este transdutor eletroacústico é formado por materiais cristalinos de efeito piezoelétrico. $O$ circuito eletrônico emite pequenos pulsos elétricos ao transdutor, excitando o cristal na sua frequência natural. Esta vibração gera ondas ultrassônicas capazes de atravessar o material em estudo (BUNGEY, 2006; ASTM C 597-02, 2003).

O aparelho geralmente possui um visor que indica a intensidade da energia refletida e a localização das interfaces. É composto por um circuito eletrônico capaz de gerar pulsos que são transformados em ondas ultrassônicas e transmitidas para o concreto, recebidas por um transdutor-receptor, amplificadas e transformadas em pulsos elétricos novamente, que permite que se meça o tempo de propagação. Pela análise destas reflexões, o usuário consegue determinar a existência ou não de descontinuidades no material (ASTM C 597-02, 2003; SANTIN, 2003).

Este tipo de ensaio pode ser visto como um dos mais propícios e com grande crescimento no ramo da engenharia para a avaliação de estruturas de concreto. Com ele consegue-se realizar um controle das variações da resistência da estrutura, que pode ser associada a mudanças das suas propriedades (LORENZI et al., 2008).

Em geral, a onda ultrassônica se propaga mais rapidamente em meios densos do que no ar. Logo, admite-se que concretos com maiores velocidades de propagação da onda ultrassônica apresentam uma maior densidade, o que de modo geral significa dizer que apresentam menor índice de vazios e, portanto, uma maior qualidade (TEODORO; CASCUDO; CARASEK, 2018). Sendo assim, a norma britânica BS EN 12504-4 Testing concrete - Determination of ultrasonic pulse velocity (BSI, 2000), apresenta uma correlação relevante, que associa os valores de velocidade do pulso ultrassônico com a qualidade do concreto, como se observa na Tabela 2. Além disso, o ultrassom também pode ser utilizado para avaliar aspectos da compacidade, bem como a presença de fissuras e falhas no concreto (MALHOTRA; CARINO, 2004).

Tabela 2 - Critérios de avaliação do concreto com base na velocidade de onda ultrassônica proposta pela norma BS EN 12504-4 (BSI, 2000)

\begin{tabular}{|c|c|}
\hline Velocidade da onda ultrassônica $(\mathbf{m} / \mathbf{s})$ & Qualidade do concreto \\
\hline$>4500$ & Excelente \\
\hline 3500 a 4500 & Bom \\
\hline 3000 a 3500 & Regular \\
\hline 2000 a 3000 & Ruim \\
\hline$<2000$ & Bom \\
\hline
\end{tabular}

Fonte: BS EN 12504-4 (BSI, 2000) 


\section{Materiais e Métodos}

A metodologia proposta para este trabalho engloba 4 etapas listadas nos subtópicos a seguir. Com o intuito de contextualizar a importância do ensaio de ultrassom como ferramenta de diagnóstico para aferir a resistência do concreto através da determinação da velocidade de propagação da onda ultrassônica foi realizada uma pesquisa bibliográfica sobre o desempenho de ensaios de ultrassom em concreto, incluindo a consulta a instruções normativas como a NBR 8802 (ABNT, 2019), NBR NM 335 (ABNT, 2012), BS 1881: 203 (BSI, 1986) e ASTM C 597- 02 (ASTM, 2003).

\subsection{Dosagem e moldagem das amostras}

As amostras de concreto utilizada neste trabalho foram produzidas com resistência característica à compressão prevista de no mínimo $30 \mathrm{MPa}$. Para a confecção dos CPs foram utilizados os seguintes materiais: cimento CP V-ARI (cimento Portland de alta resistência inicial), britas 1 e 2 de origem calcária com dimensão máxima característica de $25 \mathrm{~mm}$ e $12,5 \mathrm{~mm}$ respectivamente, e areia natural de rio, zona ótima, com módulo de finura de 2,59 .Foram produzidos 10 CPs (Figura 01) que, 24h após a moldagem foram colocados em cura úmida (Figura 02).

Figura 1 - Corpos de prova de concreto simples e corpos de prova em cura

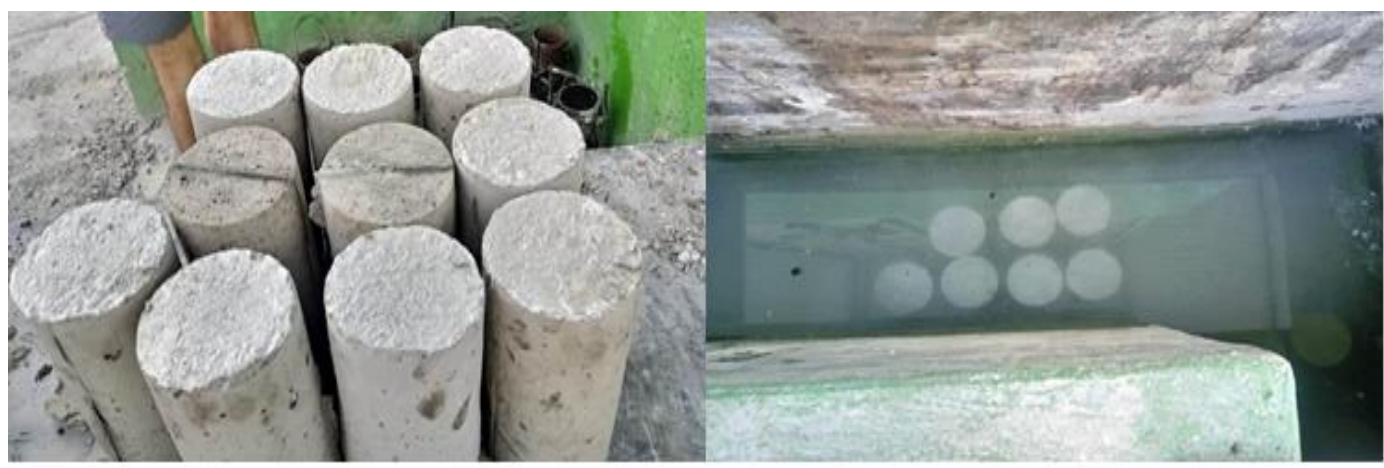

Fonte: Acervo Autores.

A resistência de dosagem obtida foi de $36,6 \mathrm{MPa}$, utilizando um desvio padrão $\left(s_{d}\right)$ de 4,0 MPa determinado conforme a condição de preparo do concreto, referente a Classe A. A relação água/cimento de 0,52 foi adotada. A proporção dos materiais agregados utilizados foi de $60 \%$ de brita 1 e $40 \%$ de brita 2 .

O traço unitário obtido é apresentado na Tabela 3 e a quantidade de material utilizada, em $\mathrm{kg}$, para a produção do concreto é apresentada na Tabela 4, para uma consistência requerida do concreto de $8 \pm 10$ $\mathrm{cm}$.

Tabela 3 - Traço unitário

\begin{tabular}{|c|c|c|c|c|}
\hline Cimento & Brita 1 & Brita 0 & Areia Natural & Água \\
\hline 1 & 1,65 & 1,10 & 1,82 & 0,45 \\
\hline
\end{tabular}

Fonte: Autores.

Tabela 4 - Quantidade de materiais do traço em kg para o volume de 34 litros

\begin{tabular}{|c|c|c|c|c|}
\hline Cimento & Brita 1 & Brita 0 & Areia Natural & Água \\
\hline $\mathrm{kg}$ & $\mathrm{kg}$ & $\mathrm{kg}$ & $\mathrm{kg}$ & $\mathrm{kg}$ \\
\hline 13,70 & 22,55 & 15,04 & 24,94 & 6,17 \\
\hline
\end{tabular}

Fonte: Autores. 


\subsection{Ensaio de Ultrassom}

A partir da moldagem dos corpos de prova o ensaio de ultrassom foi feito pelo método de medição direta (transmissão direta). Este arranjo é o mais recomendado na determinação da velocidade de propagação de ondas por um material, pois desta forma as ondas são recebidas com maior intensidade. Os transdutores devem ser posicionados em faces opostas do corpo de prova ou elemento de concreto. Foi utilizado um gel acoplante para o ensaio de ultrassom de alto desempenho para uso geral, o mesmo gel que é utilizado no equipamento para ultrassom em gestantes.

O ensaio foi realizado nas seguintes idades: 3, 5,25, 48 e 70, dias. Os ensaios foram executados em parceria com a empresa Consultare Labcon (Belo Horizonte/MG). Foram realizados dois ensaios: o não destrutivo (ultrassom) e o destrutivo (compressão axial). O ensaio não destrutivo foi feito seguindo a NBR 8802 (ABNT, 2019) que se aplica à realização de ensaios em corpos de prova e testemunhos de estruturas e também à verificação da homogeneidade do concreto de elementos estruturais, imediatamente antes da execução do ensaio de compressão axial.

\subsection{Ensaio de Compressão.}

Os ensaios de compressão foram realizados de acordo com a NBR 5739 (ABNT, 2018), logo após a execução do ensaio de ultrassom nos corpos de prova. Segundo a norma, até a idade de ensaio, os corpos de prova devem ser mantidos em processo de cura úmida ou saturados, nas condições preconizadas. Os mesmos foram mantidos em primeiro momento, no tanque da empresa CityMix (Curvelo/MG), e posteriormente, foram levados para Belo Horizonte/MG, especificamente para o tanque do laboratório LabCon, onde ficaram em cura úmida até o último ensaio.

A carga de ensaio foi aplicada continuamente e sem choques, com velocidade de carregamento de $0,45 \pm$ $0,15 \mathrm{MPa} / \mathrm{s}$. Sendo que nenhum ajuste deve ser efetuado nos controles da máquina quando o corpo de prova estiver se deformando rapidamente ao se aproximar de sua ruptura.

\section{Resultados e Discussão}

A utilização de ENDs é uma ótima alternativa, uma vez que a peça estrutural a ser avaliada não sofre dano algum ou pequenos danos facilmente reparáveis que não comprometem o seu desempenho, permitindo assim uma investigação mais ampla do concreto da estrutura.

O ensaio de US é um método eficaz de ensaio não destrutivo para controle de qualidade do concreto e detecção de danos de componentes estruturais. Em geral para uma determinada trajetória, maior tempo de deslocamento é correlacionado com concreto de baixa qualidade, anomalias e deficiências. Já o menor tempo de deslocamento é correlacionado com concreto de alta qualidade com menos anomalias.

A qualidade do concreto é geralmente especificada em termos de resistência e é, portanto, às vezes útil usar medições de VPU para obter uma estimativa da mesma. A relação entre VPU e a resistência é afetada por uma série de fatores que foram descritos anteriormente, como: idade, condições de cura, umidade, tipo de cimento. Se uma estimativa de resistência for necessária, é, portanto, essencial estabelecer uma correlação entre resistência e VPU para o tipo de concreto estudado. Esta correlação foi estabelecida experimentalmente através de ensaios em um número suficiente de amostras para cobrir a gama de resistências esperadas.

Com os dados obtidos nos ensaios de ultrassom e resistência a compressão do concreto ( $f(k j)$, foram estudadas as correlações entre a velocidade ultrassônica e a resistência à compressão do concreto, e as correlações entre a velocidade ultrassônica e o módulo de deformação do concreto. Por meio de regressão foram estudados os coeficientes de determinação $\left(R^{2}\right)$ e o de correlação $(r)$, bem como a equação de regressão aplicável à situação analisada. 
Para o desenvolvimento do experimento foram utilizados 10 corpos de prova cilíndricos de concreto com dimensões $10 \mathrm{~cm}$ (diâmetro) $\times 20 \mathrm{~cm}$ (altura). O primeiro ensaio realizado foi o de ultrassom, onde foi utilizado o aparelho de ultrassom do tipo Pundit Lab da Proceq com largura de banda de $20 \mathrm{kHz}-500 \mathrm{kHz}$. Os transdutores do aparelho foram posicionados nos eixos dos corpos de prova das faces cilíndricas oposta, passando-se gel no transdutores para que houvesse melhor contato sucessivo entre as superfícies.

Os dados fornecidos nos ensaios de ultrassom informam a velocidade de propagação da onda sônica dada em $\mathrm{m} / \mathrm{s}$ e o tempo decorrido que é informado em $\mu / \mathrm{s}$. O segundo ensaio realizado foi de resistência a compressão, onde as amostras foram colocadas em uma prensa hidráulica para rompimento. Na Tabela 5 estão indicados os valores das velocidades de propagação da onda ultrassônica e as resistências dos concretos obtidos a partir dos ensaios de compressão axial realizados nos CPs cilíndricos.

Tabela 5 - Resultados dos ensaios em concreto para diferentes idades

\begin{tabular}{|c|c|c|c|c|c|c|c|c|c|c|}
\hline Idade (dias) & 3 & 3 & 5 & 5 & 25 & 25 & 48 & 48 & 70 & 70 \\
\hline $\begin{array}{c}\text { Velocidade média } \\
\text { de propagação da } \\
\text { onda ultrassônica } \\
\text { (m/s) }\end{array}$ & 4298 & 4298 & 4391 & 4391 & 4500 & 4400 & 4666 & 4623 & 4601 & 4619 \\
\hline Carga (KN) & 167,9 & 165,7 & 203,8 & 207,6 & 299,7 & 286,9 & 333 & 313,9 & 346,4 & 340,3 \\
\hline$f_{c k j}(\mathrm{MPa})$ & 21,38 & 21,10 & 25,95 & 26,43 & 25,43 & 36,53 & 42,40 & 39,97 & 44,11 & 43,33 \\
\hline
\end{tabular}

Fonte: Autor.

Os resultados obtidos nos dois ensaios são apresentados no gráfico da Figura 2. A reta apresentada foi traçada através do método linear. A escolha da equação representativa da curva teve como parâmetro o maior valor para o coeficiente de determinação $\left(R^{2}\right)$ obtidos atraves de um estudos de regressão linear e não linear.

Figura 2: Correlação da Velocidade do Pulso Ultrassônico e da Resistência à compressão axial

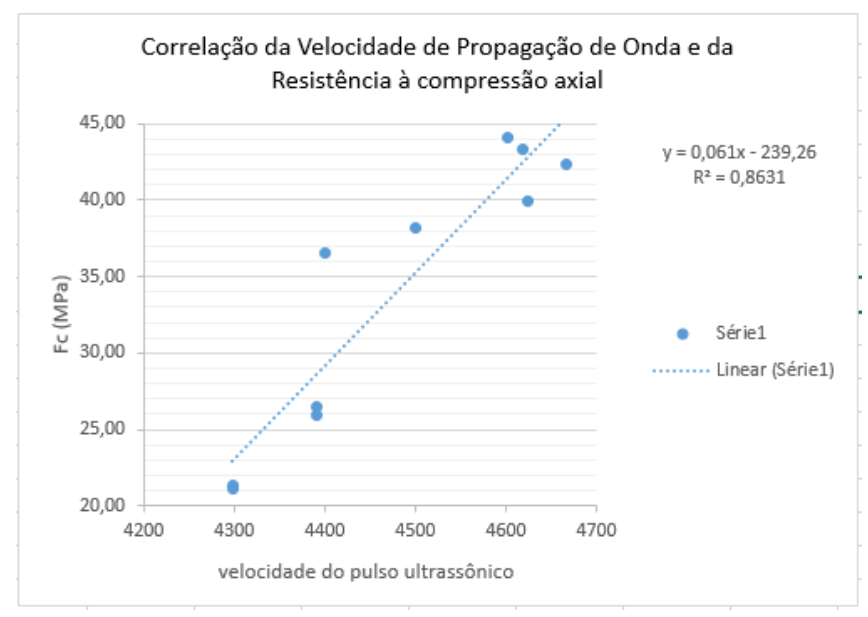

Fonte: Autor.

\section{Conclusões}

Os resultados apresentaram apenas uma correlação, isso devido a fatores que podem influenciar nessas correlações, dentre eles pode-se destacar o tipo de cimento utilizado e a relação água/cimento. A análise estatística da correlação existente entre os ensaios, mostrou que não há grandes diferenças entre os resultados. Baseado neste trabalho é interessante que futuros estudos sejam realizados utilizando esta 
mesma metodologia, porém com maior número de amostras e obtendo mais informações que possam facilitar a analise dos resultados, como o tipo de cimento, umidade do ambiente de ensaio e temperatura do local.

Com base no sresultados obtidos, o concreto posseui uma boa qualidade, já que apresentou $50 \%$ de suas velocidade de pulso acima da velocidade de $4500 \mathrm{~m} / \mathrm{s}$. Com as correlações estabelecidas neste trabalho a partir dos resultados dos ensaios realizados em partes das estruturas dos corpos de prova conclui-se que quanto maiores são os resultados de velocidade da onda ultrassônica maior é a resistência à compressão do concreto, isto se explica pelo fato de que quanto maior a compacidade do concreto, mais fácil é o fluxo da onda ultrassônica. Em relação aos coeficientes de determinação obtidos da correlação entre estas duas variáveis foram obtidos os valores $R^{2}=0,8631$, isto mostra uma forte correlação entre estas variáveis. À medida que aumentam os resultados de velocidade da onda ultra-sônica também aumenta o módulo de deformação do concreto.

\section{Agradecimentos}

Agradecemos ao Centro Federal de Educação Tecnológica de Minas Gerais - CEFET-MG pelo apoio direto e indireto a este trabalho e a empresa City Mix Concreto pela doação dos materiais utilizados e a empresa Consultare Labcon por ter cedido o espaço físico (equipamento e laboratório) para a realização dos experimentos. Agradecemos também ao LAR - Laboratório de Avaliação e Reabilitação de Ambiente Construído CEFET/MG - Campus Curvelo, pelo apoio durante o desenvolvimento do estudo.

\section{Referências Bibliográficas}

ASSOCIAÇÃO BRASILEIRA DE NORMAS TÉCNICAS - ABNT. NBR 8802: Concreto Endurecido - Determinação da velocidade propagação de onda ultrassônica. Rio de Janeiro: ABNT, 2019.

ASSOCIAÇÃO BRASILEIRA DE NORMAS TÉCNICAS - ABNT. NBR 5739: Concreto - Ensaio de compressão de corpos de prova cilíndricos. Rio de Janeiro, 2018.

AMERICAN SOCIETY FOR TESTING AND MATERIALS - ASTM. ASTM Test Designation C 579-02: Standart test method for pulsevelocity through concrete, Annual Book of ASTM Standards, Vol 04.02, West Conshohocken, PA, 2003.

BUNGEY, J. H., The of concrete testing in structures. 4 ed. London. Surrey University Press, 2006.

CASTRO, E. Estudo da resistência à compressão do concreto por meio de testemunhos de pequeno diâmetro e esclerometria. Dissertação de Mestrado em Engenharia Civil, Faculdade de Engenharia Civil da Universidade Federal de Uberlândia, Uberlândia,2009. 122p.

EVANGELISTA, A. C. J. Avaliação da resistência do concreto usando diferentes ensaios não destrutivos. 2002. 219f. Tese (Doutorado em Engenharia Civil) Universidade Federal do Rio de Janeiro, Rio de Janeiro. 2002.

MENEGHETTI, L. C. Avaliação da resistência à compressão de concretos nas primeiras idades através do ultrassom. 1999. Dissertação de Mestrado em Engenharia Civil. Universidade Federal de Santa Catarina. Florianópolis.

MALHOTRA, V.M.; CARINO, N. J. CRC handbook on nondestructive testingof concrete. Boca Raton: CRC Press, 1991. 343p.

NAIK, T. R.; MALHOTRA, V. M.; POPOVICS, J. S. The Ultrasonic Pulse Velocity Method. CRC handbook on nondestructive testing of concrete. Tarun R. Naik and V M Malhotra eds, CRC Press, pp 169-189, 2004. 